\title{
Ativação da via Enzima Conversora de Angiotensina (ECA) II/Ang 1-7/Receptor Mas como alvo farmacológico em patologias cardíacas: uma revisão sistemática
}

Way Angiotensin Converting Enzyme (ACE) II/Ang 1-7/Mas receptor activation as a pharmacological target in cardiac pathologies: a systematic review

Vía Enzima Convertidora de Angiotensina (ECA) II/Ang 1-7 /Activación del receptor pero como objetivo farmacológico en patologías cardíacas: una revisión sistemática

Igor Matheus Oliveira da Silva ORCID: https://orcid.org/0000-0002-8627-4015 Faculdade de Ciências Humanas, Exatas e da Saúde do Piauí, Brasil Instituto de Educação Superior do Vale do Parnaíba, Brasil E-mail: igor_matheussilva@outlook.com Júlia de Sousa Caroba

ORCID: https://orcid.org/0000-0001-7027-3686 Faculdade de Ciências Humanas, Exatas e da Saúde do Piauí, Brasil Instituto de Educação Superior do Vale do Parnaíba, Brasil E-mail: juliacaroba@ hotmail.com Matheus Almeida Thorpe

ORCID: https://orcid.org/0000-0002-1798-3889 Faculdade de Ciências Humanas, Exatas e da Saúde do Piauí, Brasil Instituto de Educação Superior do Vale do Parnaíba, Brasil E-mail: matheusthorpe@gmail.com

Luan Kelves Miranda de Souza ORCID: https://orcid.org/0000-0002-8019-4022 Faculdade de Ciências Humanas, Exatas e da Saúde do Piauí, Brasil Instituto de Educação Superior do Vale do Parnaíba, Brasil E-mail: luankelves11@gmail.com

\begin{abstract}
Resumo
Objetivo: Compreender a ativação da via ECA II/ Ang 1-7/Receptor Mas como alvo farmacológico em patologias cardíacas. Metodologia: Trata-se de uma revisão de literatura sistemática, pesquisadas nas bases de dados EMBASE, MEDLINE e SCIELO, que para extração dos dados primários e transformação em dados secundários, foi utilizado o protocolo PRISMA. Resultados: O Sistema Renina Angiotensina é um integrante imprescindível para o adequado funcionamento fisiológico e homeostasia do sistema cardiovascular. Sendo divido em dois eixos, o clássico, que é o usado na atualidade na farmacoterapia da hipertensão e doenças cardiovasculares. E o eixo não clássico que através da via da enzima conversora de angiotensina II (ECA II), angiotensina (1-7) e receptor Mas, que tem demonstrado importante efeito vasodilatador e cardioprotetor. Demonstram um desfecho promissor e reforçam a ação efetiva do MasR como vasodilatador, cardioproteção e redução da remodelagem miocárdica. Considerações Finais: O eixo não clássico possui potencial no desenvolvimento de drogas para expansão de novas estratégias farmacológicas para as doenças cardiovasculares.
\end{abstract}

Palavras-chave: Doenças cardiovasculares; Angiotensinas; Sistema renina-angiotensina; Receptores de angiotensina; Desenvolvimento de medicamentos.

\begin{abstract}
Objective: To understand the activation of the ECA II / Ang 1-7 / Receptor Mas route as a pharmacological target in cardiac pathologies. Methodology: This is a systematic literature review, researched in the EMBASE, MEDLINE and SCIELO databases, which used the PRISMA protocol to extract primary data and transform it into secondary data. Results: The Renina Angiotensin System is an essential component for the proper physiological functioning and homeostasis of the cardiovascular system. Being divided into two axes, the classic, which is currently used in the pharmacotherapy of hypertension and cardiovascular diseases. It is the non-classical axis that, via the angiotensin II converting enzyme (ACE II), angiotensin (1-7) and Mas receptor pathway, has demonstrated an important vasodilator and cardioprotective effect. They demonstrate a promising outcome and reinforce the effective action of MasR as a vasodilator, cardioprotection and reduction of myocardial remodeling. Final Considerations: The non-classical axis has potential in the development of drugs to expand new pharmacological strategies for cardiovascular diseases.
\end{abstract}

Keywords: Cardiovascular diseases; Angiotensins; Renin-angiotensin system; Receptors, Angiotensin; Drug development. 


\begin{abstract}
Resumen
Objetivo: Comprender la activación de la ruta ECA II / Ang 1-7 / Receptor Mas como diana farmacológica en patologías cardíacas. Metodología: Se trata de una revisión sistemática de la literatura, investigada en las bases de datos EMBASE, MEDLINE y SCIELO, que utilizó el protocolo PRISMA para extraer datos primarios y transformarlos en datos secundarios. Resultados: El Sistema Renina Angiotensin es un componente esencial para el correcto funcionamiento fisiológico y homeostasis del sistema cardiovascular. Estando dividido en dos ejes, el clásico, que actualmente se utiliza en la farmacoterapia de la hipertensión y las enfermedades cardiovasculares. Es el eje no clásico que, a través de la enzima convertidora de angiotensina II (ECA II), la vía del receptor de angiotensina (1-7) y Mas, ha demostrado un importante efecto vasodilatador y cardioprotector. Demuestran un resultado prometedor y refuerzan la acción eficaz de MasR como vasodilatador, cardioprotector y reductor del remodelado miocárdico. Consideraciones finales: El eje no clásico tiene potencial en el desarrollo de fármacos para expandir nuevas estrategias farmacológicas para las enfermedades cardiovasculares.
\end{abstract}

Palabras clave: Enfermedades cardiovasculares; Angiotensinas; Sistema renina-angiotensina; Receptores de angiotensina; Desarrollo de medicamentos.

\title{
1. Introdução
}

As doenças cardiovasculares (DCV) representam um expressivo desafio à saúde mundial decorrente de sua elevada prevalência e por ser uma das principais causas de morte no mundo (Tufik et al., 2017). As DCV foram responsáveis por 17,7 milhões de mortes em 2015, representando 31\% das mortes em nível global (OPAS/OMS, 2017). Com perspectiva de progressão ascendente e apresenta um importante problema a sua pública devido a sua associação com incapacitação e alta mortalidade.

A fisiopatologia que envolve seu desenvolvimento está relacionada a alterações progressivas na anatomia, fisiologia cardíaca e vascular, que sofrem interações com a exposição cumulativa a fatores de risco (Merz \& Cheng, 2016). Dentre os fatores determinantes que favorecem as DCVs, pode-se citar, diabetes mellitus, nutrição, tabagismo, estresse, hipertensão, e alterações no ritmo circadiano (Zhang et al., 2018). Mas o entendimento da fisiopatologia desencadeante dessa morbidade ainda não está totalmente elucidado, devido à complexidade das interações celulares envolvidas.

O Sistema Renina Angiotensina (RAS) é um integrante imprescindível para o adequado funcionamento fisiológico e a homeostasia do sistema cardiovascular (Cole-Jeffrey et al., 2015). Esse sistema realiza função sistêmica significativa no equilíbrio pressórico e manutenção do equilíbrio hidroeletrolítico, e localmente promove em órgãos e tecidos, crescimento e diferenciação celular (Pinter \& Jain, 2017). Tendo funções significativas como mediador de arteriosclerose, hipertensão arterial, fibrose miocárdica, hipertrofia ventricular esquerda e insuficiência cardíaca congestiva (Wang et al., 2017).

O RAS tem sido estudado amplamente, e a recente descoberta de novos componentes desse sistema alterou a forma que é entendido no âmbito fisiológico. Sendo divido em dois eixos, o clássico, que é o usado atualmente na farmacoterapia da hipertensão e doenças cardiovasculares (Povlsen et al., 2020). E o eixo não clássico tem demonstrando importante efeito vasodilatador e cardioprotetor (Mendoza-Torres et al., 2015).

O eixo clássico é a via que envolve a enzima conversora de angiotensina I (ECAI), que é responsável pela conversão da angiotensina 1 (Ang 1) em angiotensina 2 (Ang 2) (Qaradakhi et al., 2020). A Ang II é o principal componente efetivo desse sistema, como potente poder vasoconstritor, ação pró-inflamatória e estimula a secreção adrenal de aldosterona. Para desempenhar sua ação, liga-se principalmente ao receptor acoplado a proteína G, o RAT2 (Patel et al., 2017).

Com o evidente e fundamental papel do eixo clássico na regulamentação do controle pressórico, servindo assim, de base para primeira linha de tratamento da hipertensão arterial. As estratégias farmacológicas atuais atuam em diferentes locos desse eixo, tendo efeito não apenas no controle pressórico, mas também, na redução da remodelagem ventricular e prevenção da insuficiência cardíaca (Povlsen et al., 2020). As drogas que se baseiam nesse eixo tem ação de diminuir o efeito do mesmo, tais como, inibidores da enzima conversora de angiotensina e antagonista dos receptores de angiotensina 2 (Ferrario et al., 2017). 
As drogas que atuam no SRAA e que possuem relevante espaço nas estratégias farmacológicas atuais, são os inibidores da enzima conversora de renina (IECA), bloqueadores dos receptores AT1 (BRA), antagonistas de receptores mineralocorticóides (MRAs) (Wang et al., 2017). No entanto, abordagens terapêuticas atuais também se baseiam na diminuição de fatores de risco, tais como, modificação da dieta, instituição de exercício físico e interrupção do tabagismo e etilismo, e farmacoterapia (Costantino et al., 2015).

O não clássico é o eixo vasorelaxante, que possui papel de proteção cardiovascular em contraponto a ação exacerbada do eixo clássico (Santos et al., 2018). Sua ação se fundamenta na via da enzima conversora de angiotensina II (ECA II), que converte Ang 2 em angiotensina (1-7) e Ang 1 em angiotensina (1-9), ligando-se aos receptores Mas (MasR) e RAT2, respectivamente, mas podendo ser convertido por outras enzimas (Awwad et al., 2019). O Ang (1-7) heptapeptídeo vasodilatador que é um agonista específico do receptor Mas (MasR), que tem a funções do MasR neutralizam os efeitos adversos da via clássica (Silva et al., 2016)

De acordo com a crescente prevalência e mortalidade das doenças cardiovasculares e a dificuldade na efetividade terapêutica com as drogas existentes e descoberta de nova via do RAS, levam a novos esforços no desenvolvimento de drogas com potencial terapêutico mais eficiente (Ferrario et al., 2017). Busca-se investigar a eficácia da via ECAII, pela angiotensina (1-7) e o MasR, como nova estratégia no combate às doenças cardiovasculares (Riet et al., 2015). Com isso, o objetivo do trabalho é compreender a ativação da via ECA II/ Ang 1-7 / receptor Mas como alvo farmacológico em patologias cardíacas e verificar os efeitos cardioprotetores do eixo não clássico, comparando o papel desse eixo com o eixo clássico na homeostase.

\section{Metodologia}

Trata-se de uma revisão de literatura sistemática qualitativo, na qual foram pesquisadas publicações científicas nas bases de dados Excerpta Medica Database (EMBASE), Medical Literature Analysis and Retrievel System Online (MEDLINE) e Scientific Electronic Library Online (SCIELO). Foram utilizados os descritores em português "doenças cardiovasculares", "angiotensina", "sistema renina-angiotensina", "cardioproteção", "desenvolvimento de medicamentos". Em inglês os descritores foram "cardiovascular diseases", "angiotensin", "renin-angiotensin system", " cardioprotection", "drug development". Pesquisados de forma associada, a unir utilizando o operador booleano: AND.

Para extração dos dados primários e transformação em dados secundários, foi utilizada a lista de recomendação do protocolo PRISMA, que visa ajudar os autores a melhorarem o relato de revisões sistemáticas e meta-análise. As referências dos artigos encontrados passaram por uma revisão manual para verificação de relevância e inclusão de estudos. (Moher et al., 2015)

Dos registros selecionados, foram analisados forma e local de intervenção, presença de um grupo controle ou procedimento simulado, o tipo de estudo, ação no sistema cardiovascular, mecanismo de ação, medidas de resultado (avaliar a diminuição dos níveis pressóricos e alterações com potencial de cardioproteção) e possíveis efeitos deletérios da estimulação do eixo não clássico.

\section{Resultados e Discussão}

Um total de 45 registros foram identificados pela triagem inicial após busca nas bases de dados. Após a revisão das referências foram adicionados 01 registro. Os títulos e resumos foram analisados por todos os integrantes individualmente. Destes trabalhos, apenas 06 foram incluídos para compor a pesquisa, os quais tiveram todo seu texto lido para garantir a elegibilidade. Dos selecionados dividem-se em trabalhos realizados com animais (três em camundongos, um em ratos e um 
modelo canino) e trabalhos realizados com seres humanos (uma publicação). A (Figura 1) a seguir, traz a descrição destes artigos.

O (Quadro 1), é encontrado a forma cronológica, os resultados extraídos dos artigos utilizados para a investigação. Bem como os dados retirados de cada estudo, como, autores, ano, amostra, tipo de estudo e desfecho.

Figura 1 - Delineamento do estudo nas bases de dados.

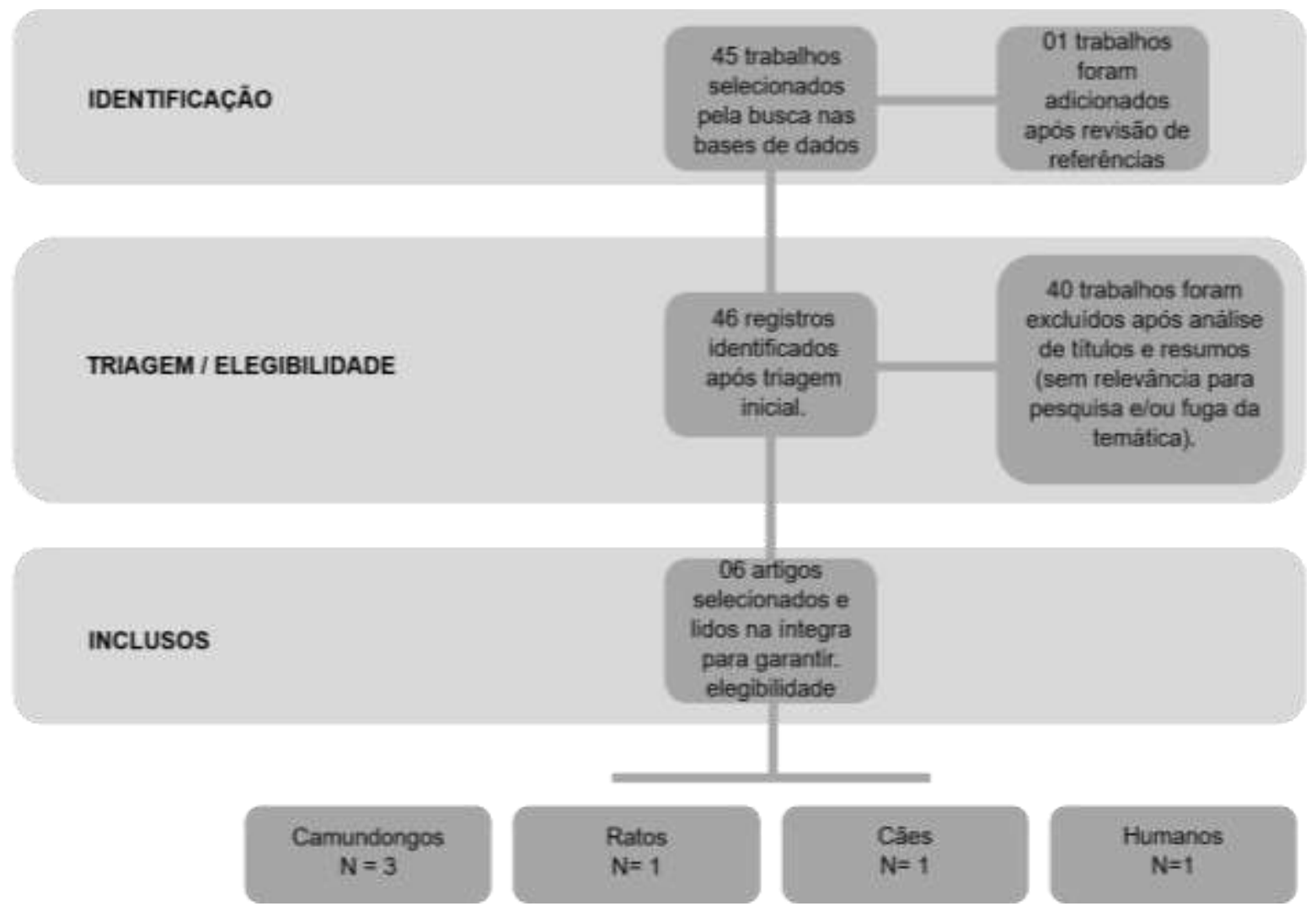

Fonte: Silva e Souza (2020).

O (Quadro 1), é encontrado na forma cronológica, os resultados extraídos dos artigos utilizados para a investigação. Bem como os dados retirados de cada estudo, como, autores, ano, amostra, tipo de estudo e desfecho. 
Quadro 1 - Artigos incluídos no estudo nas referidas bases de dados MEDLINE, LILACS e SCIELO 2019.

\begin{tabular}{|c|c|c|c|c|c|}
\hline $\mathbf{N}^{\circ}$ & AUTOR & ANO & AMOSTRA & $\begin{array}{l}\text { TIPO DE } \\
\text { ESTUDO }\end{array}$ & DESFECHO \\
\hline 1 & Patel et al. & 2015 & $\begin{array}{l}\text { Camundongo } \\
\qquad \mathrm{N}=06\end{array}$ & $\begin{array}{l}\text { Estudo } \\
\text { prospectivo }\end{array}$ & $\begin{array}{l}\text { Redução da hipertrofia concêntrica e disfunção diastólica com função } \\
\text { sistólica preservada. }\end{array}$ \\
\hline 2 & Basu et al. & 2017 & $\begin{array}{l}\text { Humanos } \\
\mathrm{N}=101\end{array}$ & $\begin{array}{l}\text { Estudo } \\
\text { prospectivo }\end{array}$ & $\begin{array}{l}\text { Nível plasmático reduzido de Ang- (1-7) está associado a IC, pelo aumento } \\
\qquad \text { de Ang } 2 . \\
\text { Aumento de ACE II normalizou efetivamente Ang } 2 \text { elevado. }\end{array}$ \\
\hline 3 & Hao et al. & 2018 & $\begin{array}{l}\text { Camundongo } \\
\qquad \mathrm{N}=39\end{array}$ & $\begin{array}{l}\text { Estudo } \\
\text { prospectivo }\end{array}$ & Ação protetora do aneurisma de aorta, pela inibição da resposta inflamatória. \\
\hline 4 & Meems et al. & 2019 & $\begin{array}{l}\text { Cães } \\
\mathrm{N}=16\end{array}$ & $\begin{array}{l}\text { Estudo } \\
\text { prospectivo }\end{array}$ & $\begin{array}{c}\text { Redução da pressão arterial média, diminuição da resistência vascular } \\
\text { sistêmica e promove diurese e natriurese. }\end{array}$ \\
\hline 5 & Liao et al. & 2019 & $\begin{array}{l}\text { Ratos } \\
\mathrm{N}=24\end{array}$ & $\begin{array}{l}\text { Estudo } \\
\text { prospectivo }\end{array}$ & $\begin{array}{l}\text { Efeito anti-hipertensivo e diminuição da disfunção endotelial, através da } \\
\text { vasodilatação. } \\
\text { Inibição da formação de lesões ateroscleróticas e no aumento da estabilidade } \\
\text { da placa. }\end{array}$ \\
\hline 6 & $\begin{array}{l}\text { Stegbauer et } \\
\text { al. }\end{array}$ & 2019 & $\begin{array}{l}\text { Camundongo } \\
\qquad \mathrm{N}=20\end{array}$ & $\begin{array}{l}\text { Estudo } \\
\text { prospectivo }\end{array}$ & $\begin{array}{l}\text { Dificulta a formação de lesões ateroscleróticas e o desenvolvimento e } \\
\text { gravidade dos aneurismas da aorta abdominal. }\end{array}$ \\
\hline
\end{tabular}

Fonte: Silva e Souza (2020).

O sistema renina angiotensina (RAS) é um componente fundamental para o sistema cardiovascular, fisiologia e homeostasia (Colafella et al., 2016). Visto que, possui influência direta na regulação do balanço hidroeletrolítico, pressão arterial, tônus muscular e remodelagem miocárdica e endotelial (Qaradakhi et al., 2020). A expressão local em órgãos desse sistema promove crescimento, diferenciação e inflamação, através de uma série de complexos moleculares e celulares.

Em condições fisiológicas normais existe o equilíbrio entre dois eixos, o clássico (canônico) e não clássico, que auxiliam na homeostase cardiovascular (Colafella et al., 2019). A desregulamentação deste sistema implica na gênese da fisiopatologia de inúmeras doenças cardiovasculares, renais, pulmonares e até cerebrais (Wang et al., 2019). O RAS disfuncional possui íntima relação com o desencadeamento de alterações da homeostase e fisiologia desencadeante de doenças cardiovasculares, pois modulo diversos mecanismos necessários para o funcionamento desse sistema (Figura 2). 
Figura 2 - Contra regulação dos eixos clássico e alternativo.

PATOGÊNESE DAS DCV

- VAsoconstriçäo

- estResse oxidativo

- PRÓ - FIBRÓTICO

- PRÓ - INFLAMATÓRIO

- PROLIFERATIVO
CARDIOPROTEÇÄO

- VASODILATACĀo

- ANTI - ESTRESSE oXIDATIVO

- ANTI - FIBRÓTICO

* ANTI - INFLAMATÓRIO

+ ANTIPROLIFERATIVO

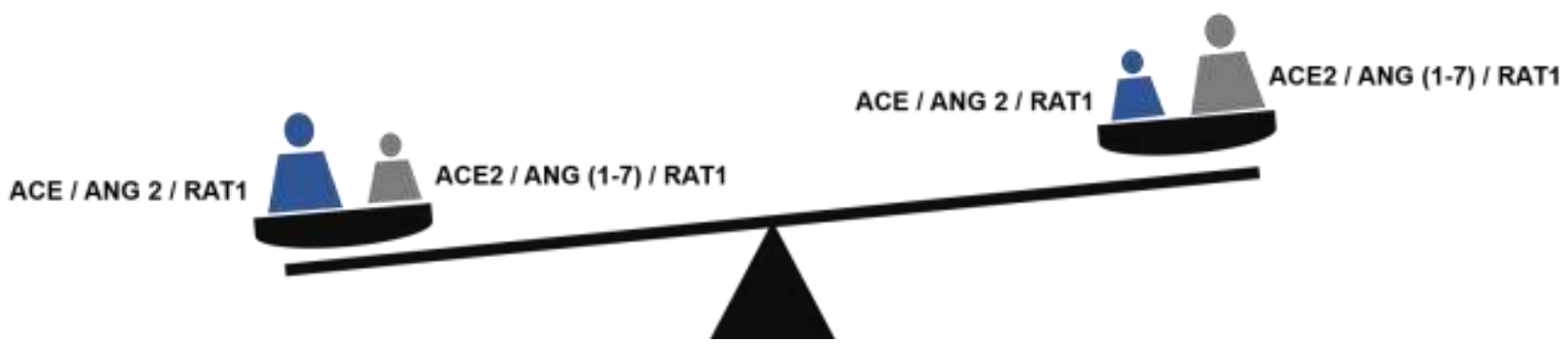

Fonte: Stegbauer et al. (2019).

\section{Eixo clássico (canônico)}

O clássico, pode ser descrito como o eixo da enzima de conversão da angiotensina I (ECA I) (Povlsen et al., 2020). A ECA é incumbida de converter a angiotensina 1 (Ang 1) em angiotensina 2 (Ang 2), que por sua vez, desempenha ação através de dois receptores distintos acoplados a proteína G: RAT1 e RAT2 (Patel et al., 2017) (figura 3). O RAT1 é responsável por grande parte dos efeitos lógicos e patológicos da Ang 2, sendo esse receptor, extensivamente expresso nas células cardiovasculares.

A ativação do RAT1 por Ang 2 induz muitos efeitos intracelulares, envolvendo fosfolipase C (PLC), Ca2 +, fosfolipase D (PLD), fosfolipase A2 (PLA2), adenilato ciclase, MAPK, transdutor de sinal de Janus quinase (JAK), ativador de transcrição (STAT) e fosfato de dinucleotídeo de nicotinamida adenina (NADPH) (Montezano et al., 2014). Sendo responsável, em estado normal, pela regulação da fisiologia sistêmica e da homeostase, através da vasoconstrição, indução a ingestão hídrica e retenção de sódio (Wang et al., 2017).

Em condições disfuncionais está associado ao aumento do estresse oxidativo, apoptose, fibrose e inflamação, favorecendo o desenvolvimento de doenças cardiovasculares (Tóth et al., 2018). Já os efeitos do RAT2 se opõem aos do RAT1, onde sua ativação estimula a vasodilatação, efeitos anti-inflamatórios e anti proliferativos. Podendo assim, modular doenças cardiovasculares, progressão das ateroscleroses, regular a PA, fibrose e morte celular (Mendoza-Torres et al., 2015).

A ativação do RAT2 promove a ativação da cascata de sinalização de óxido nítrico (NO), bradicinina e o monofosfato de guanosina cíclico (cGMP), que podem ocorrer concomitantemente ou de forma independente uma da outra, como a cGMP que pode atuar aumentando a bradicinina para efetuar sua cascata ou sem depender da bradicinina (Azushima et al., 2020).

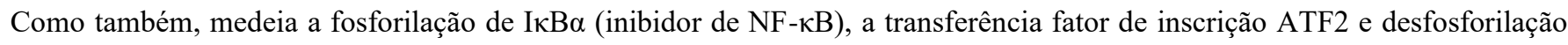
de JNK, p38, ERK1 / 2 e STAT3 (Forrester et al., 2018). O que lhe confere efeitos antagonistas às ações deletérias impulsionados pela interação do Ang2 com o RAT1.

\section{Eixo não clássico (alternativo)}

Os principais componentes dessa via alternativa com ação contra regulatória da via ECA/ Ang 2/ RAT1 são a ECA II, angiotensina (1-7), receptor, mas, receptor D acoplado à proteína G relacionado com Mas (MrgD) e peptídeo Alamandina 
(Hrenak et al., 2016). A ECA II é uma enzima ligada a membrana celular, que pode ser encontrada no tecido cardiovascular, hepático, renal e em vasos cerebrais, podendo também, está presente solúvel no plasma (Mendoza-Torres et al., 2015).

A renina cliva o angiotensinogênio no decapeptídeo angiotensina I, que por sua vez, pode ser convertida em angiotensina II. Essa ação ocorre através de três enzimas; ECA I, quimase e catepsina, que são capazes de remover o dipeptídeo histidina-leucina da angiotensina I. Mas o angiotensinogênio pode ser convertido diretamente em angiotensina II, isso é mediado pelas enzimas tonina e catepsina G, que por sua vez, nos receptores RAT1 e RAT2.

A ECA II possui alta afinidade a Ang2, convertendo o mesmo em Ang (1-7), mas também atua sobre a Ang 1 formando a Ang (1-9), ambas do eixo não clássico (South et al, 2019). A Ang (1-7) pode ser originada através de três diferentes vias: Ang (1-9) clivada em Ang (1-7) pela ECA ou a endopeptidase neutra (NEP), Ang I dando origem à Ang (1-7) pela ação enzimática da NEP, prolil-endopeptidase (PEP), prolil oligopeptidase (POP) ou Thimet Oligopeptidase, e Ang 2 convertida em Ang (1-7) pela clivagem promovida pela ECA II, a prolil-endopeptidase (PEP) ou a prolil-carxipeptidase (PCP) (figura 3).

No entanto, a via de maior expressão é a que ocorre pela ação da ECA II, por ser a principal forma de formação de Ang 2, como também, pela ação direta de conversão do Ang 2 em Ang (1-7), como indiretamente pela clivagem de Ang $1 \mathrm{em}$ (1-7). Onde a redução da função e/ou da biodisponibilidade da enzima ECA II resulta no aumento dos níveis de Ang2. Assim, favorecendo a gênese das doenças cardiovasculares pelo desequilíbrio do contrabalanceamento que ocorre entre os dois eixos do RAS (Basu et al., 2017).

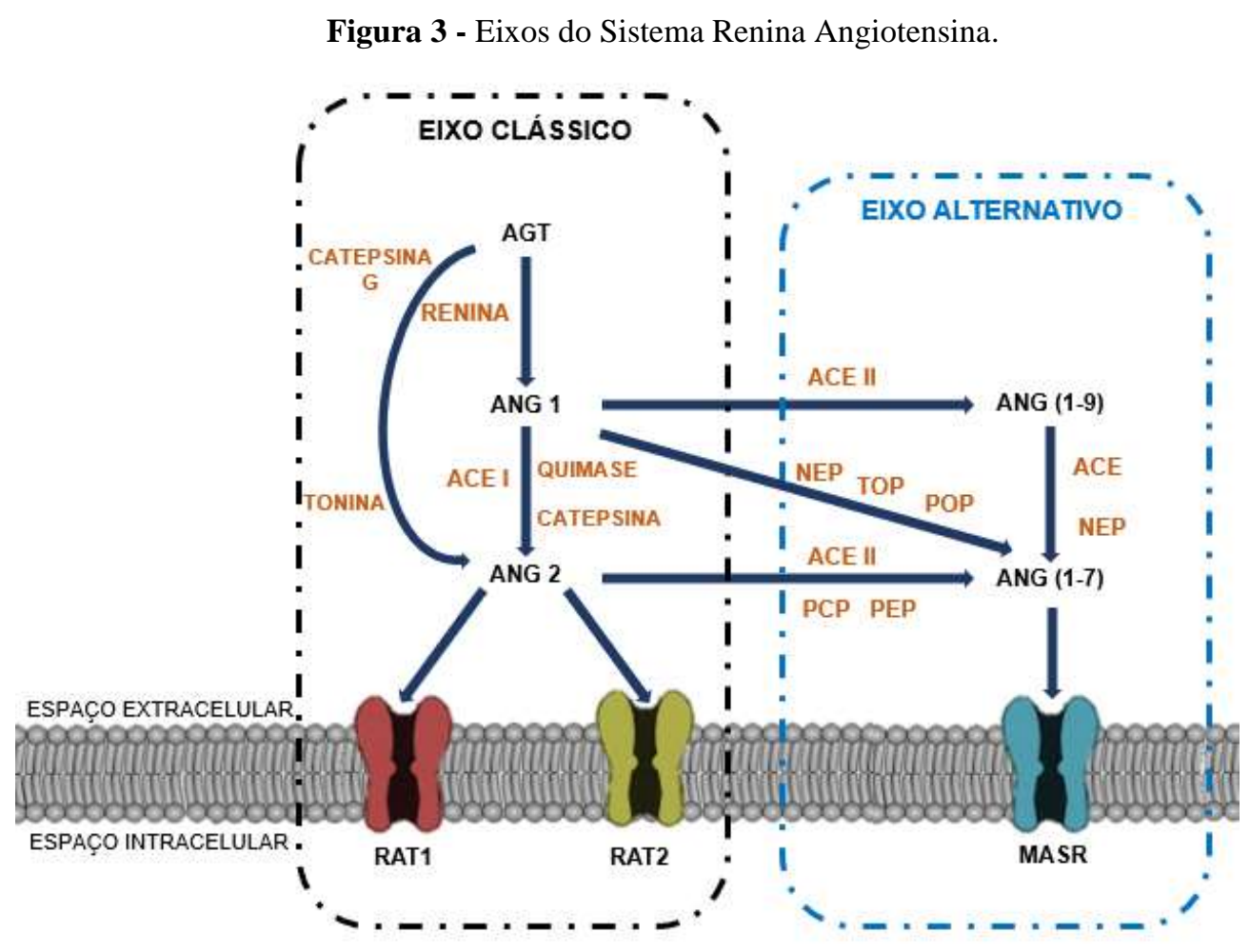

Fonte: Arroja et al. (2016).

Basu et al., (2019) através da aferição plasmática de angiotensina em humanos com Insuficiência Cardíaca (IC) crônica e aguda, observou níveis 8 a 10 vezes maiores de Ang 2. Como também, níveis mais baixos de Ang- (1-7), e a razão Ang (1-7) / Ang 2 reduzida foi associada a sintomas de IC mais severos e maior tempo de internação hospitalar. Quando 
aumentado ECA II normalizou os níveis de Ang 2, aumentando os níveis de Ang (1-7) e Ang (1-9). Demonstrando que níveis elevados de Ang 2 e baixos níveis de Ang (1-7) e Ang (1-9) estão associados ao desenvolvimento e severidade de IC.

Liao et al., (2019), em seu artigo evidencia a via ECA II / Ang (1-7) / MasR promove a diminuição do nível de Ang 2, através da utilização de um tripeptídeo derivado da ervilha, o LRW, em ratos. Constatou ao estimular a via alternativa do RAS, obteve efeitos anti-hipertensivos e da diminuição da disfunção endotelial. Esses efeitos benéficos podem ser explicados, respectivamente, pela vasodilatação e a ação anti-inflamatória.

O efeito anti-inflamatório observado no artigo de Liao et al., (2019) é oriundo da inibição na via de sinalização EROsNF-kB. Onde espécies reativas de oxigênio (EROs) acionam a enzima c-Jun N-terminal Cinase (JNK) que leva a ativação da Fator Nuclear Kappa $\beta$ (NF-kB). Paralelo a isso, o inibidor desse sistema, o ikB $\alpha$ é degradado pela cinase do Inibidor no Fator de Transcrição NF-kB (iKK) e a fosforilação de p50 e p65 (Tobón, et al., 2014).

Esse processo repercute no núcleo celular, onde promove a transcrição de mediadores pró-inflamatórios, como interleucina 6 e 10, e o Fator de Necrose Tumoral alfa (TNF- $\alpha$ ), responsáveis por acarretar disfunção endotelial (Cildir et al., 2016). Ao levar a restauração do ikBa, reduz a fosforilação do p50 e p65, e a inibição da via de sinalização EROs-NF-kB, evidencia a ação cardioprotetora do eixo ACE II/Ang (1-7) /MasR (Ferreira et al., 2016).

Também foi observado no trabalho de Liao et al., (2019), que a estimulação crônica de Ang (1-7) resultou na expressão desregulada de MMP9 na via de sinalização de ERK1 / 2 MAPK. Promovendo subsequente inibição da formação de lesões ateroscleróticas e o aumento da estabilidade da mesma. As proteínas MAPKs (Proteínas Quinases Ativadas por Mitogênio) são divididos em três subfamílias: ERK (Cinase Regulada Por Sinal Extracelular); JNK e p38 (Cargnello e Roux, 2011).

Essas vias coadjuvam inúmeros processos biológicos essenciais, tais como: controle do ciclo celular, proliferação de células, apoptose, ativação do sistema imune e proteção contra o estresse oxidativo. Onde, Patel et al., (2015) em seu estudo verificou que a inibição da Ang (1-7) e/ou aumento de Ang 2 em camundongos, levou a fosforilação de ERK1 / 2, JNK1 / 2 e p38 no miocárdio e a ativação da MMP2. Resultando no aumento da fibrose intersticial, remodelação miocárdica adversa e a insuficiência cardíaca.

No qual, o uso do antagonista da Ang (1-7), o A779, assim como, a infusão de Ang2, impulsionou a disfunção diastólica e precipitou a disfunção sistólica pela fração de ejeção reduzida e encurtamento fracionário. Na mesma proporção que a redução de Ang2 pelo aumento de ECA II e bloqueio do antagonismo de Ang 1-7, impede o estresse oxidativo do miocárdio mediado por Ang2, ativação MMP2 e impedindo os efeitos hipertróficos e pró-fibróticos. Consolidando os efeitos cardioprotetores mediados pela angiotensina (1-7) e a ECA II.

Hao et al., (2018) também comprova que a super expressão da ECA II regula as vias de sinalização ERK e NF-אB, inibindo a expressão de MMP9 e MMP2, como também, suprimiu a expressão de MCP-1, reduzindo a infiltração de macrófagos. Em que, o aumento da expressão de ECA II na aorta abdominal de camundongos demonstrou-se como um fator de proteção contra os aneurismas, por sua ação anti-inflamatória. Já o aumento de Ang 2 favoreceu o desenvolvimento de Aneurisma na Aorta Abdominal (AAA).

Através dos estudos de Liao et al., (2019), Patel et al., (2015) e Hao et al., (2018) que expõem os efeitos positivos da via ECA II / Ang (1-7)/ MasR sobre as cascatas de sinalização das vias que envolvem as proteínas MAPKs. Apresentam e reforçam as ações anti-inflamatória, anti-fibrótica e anti-apoptótica do eixo não clássico, o que lhe confere capacidade de mediar a redução da remodelagem e proliferação cardiovascular, diminuindo danos ao sistema.

Em sua pesquisa, Meems et al., (2019) através antagonismo de MasR com o MasR-i em cães, Ang 1-7 não reduziu a pressão arterial média, resistência vascular sistêmica e atenuou a diurese e a natriurese. Contudo, quando MasR não foi inibido, possibilitou as ações da Ang (1-7). Esses resultados apontam que por meio da atividade da via alternativa em diversos 
processos do sistema cardiovascular, lhe atribui importante efeito anti-hipertensiva. Esse efeito quando perpetuado, a suspensão da gênese de alterações anátomo-fisiológicas que levam a DCV.

Stergbauer et al., (2019) em seu estudo, verifica os efeitos da deficiência do MasR com o aumento da Ang2 em camundongos, e revela que favoreceu o surgimento das lesões ateroscleróticas e o aumentou área superficial, corroborando com resultados de Liao, et al., (2019). Evidenciou também, que a deficiência de MasR propicia o aumento da incidência, gravidade e o risco de ruptura de AAA, reafirmando o desfecho do Hao, et al., (2018).

Com sua pesquisa, Stergbauer et al., (2019), confirma os efeitos protetores do MasR, em evitar e diminuir a gravidade das enfermidades vasculares. Bem como, potencialmente dificultar eventos isquêmicos decorrentes das lesões ateroscleróticas, podendo atuar não apenas na prevenção, mas atuar também como tratamento e diminuição de agravos. No entanto, vale ressaltar a necessidade de mais estudos para melhor compreensão dos mecanismos envolvidos.

\section{Conclusão}

A inibição da via clássica está entre as principais estratégias no tratamento da Hipertensão Arterial e algumas doenças cardiovasculares. Mas por possuir limitações significativas são necessárias associações medicamentosas, dificultando o sucesso clínica e a adesão. Desse modo, é imprescindível a necessidade de novas drogas mais eficazes, que trate e previna doenças cardiovasculares mais efetivamente. A via ACE II / Ang- (1-7) / MasR por exerce ação anti-inflamatórios, fibrótico, apoptótica e hipertensivas, contendo a gênese das alterações anátomo-fisiológica, demonstrando como potencial efeito cardioprotetor. As evidências apresentadas aqui acrescem que a ativação da via alternativa do RAS possui potencial promissor como alvo farmacológico, para o desenvolvimento de futura e importante estratégia terapêutica para doenças cardiovasculares. Com a melhoria na eficácia das estratégias terapêuticas e diminuição dos efeitos deletérios das mesmas, pode-se diminuir a invalidez e mortalidade decorrente das afecções cardiovasculares, com consequente diminuição dos gastos públicos.

Contudo, vale ressaltar que na construção do presente trabalho foi levantado alguns aspectos relevantes para um abordagens de novos trabalhos, tais como: Estudos para melhor compreender o mecanismo exato de ação da via ACE II / Ang(1-7) / MasR e suas interações locais e sistêmicas, trabalhos para elucidar as implicações da via alternativas sobre as cadeias do ciclo celular, com maior gama para se obter o seus efeitos, de forma mais detalhados; ensaios para avaliar os efeitos dos agonistas da via ACE II / Ang- (1-7) / MasR, como o AVE0991, CGEN-856S, HPßCD- Ang-(1-7), Cyclic Ang-(1-7) e RB150/QGC001.

\section{Referências}

Arroja, M. M. C., Reid, E., \& McCabe, C. (2016). Therapeutic potential of the renin angiotensin system in ischaemic stroke. Experimental \& translational stroke medicine, $8(1), 1-14$.

Awwad, Z. M., El-Ganainy, S. O., ElMallah, A. I., Khattab, M. M., \& El-Khatib, A. S. (2019). Telmisartan and captopril ameliorate pregabalin-induced heart failure in rats. Toxicology, 428, 152310.

Azushima, K., Morisawa, N., Tamura, K., \& Nishiyama, A. (2020). Recent research advances in renin-angiotensin-aldosterone system receptors. Current hypertension reports, 22(3), 1-10.

Basu, R., Poglitsch, M., Yogasundaram, H., Thomas, J., Rowe, B. H., \& Oudit, G. Y. (2017). Roles of angiotensin peptides and recombinant human ACE2 in heart failure. Journal of the American College of Cardiology, 69(7), 805-819.

Cargnello, M., \& Roux, P. P. (2011). Ativação e função das MAPKs e seus substratos, as proteínas quinases ativadas por MAPK. Microbiology and molecular biology reviews, 75 (1), 50-83.

Cildir, G., Low, K. C., \& Tergaonkar, V. (2016). Noncanonical NF-кB signaling in health and disease. Trends in molecular medicine, $22(5), 414-429$.

Colafella, K. M. M., Hilliard, L. M., \& Denton, K. M. (2016). Épocas no equilíbrio depressor / pressor do sistema renina-angiotensina. Clinical Science, 130 (10), 761-771. 
Colafella, K. M. M., Bovée, D. M., \& Danser, A. J. (2019). O sistema renina-angiotensina-aldosterona e seus alvos terapêuticos. Pesquisa experimental do olho , 186,107680 .

Cole-Jeffrey, C. T., Liu, M., Katovich, M. J., Raizada, M. K., \& Shenoy, V. (2015). ACE2 and microbiota: emerging targets for cardiopulmonary disease therapy. Journal of cardiovascular pharmacology, 66(6), 540.

Costantino, S., Paneni, F., \& Cosentino, F. (2016). Ageing, metabolism and cardiovascular disease. The Journal of physiology, 594(8), $2061-2073$.

e Silva, A. C. S., \& Teixeira, M. M. (2016). ACE inhibition, ACE2 and angiotensin-(1 7$)$ axis in kidney and cardiac inflammation and fibrosis. Pharmacological research, 107, 154-162.

Ferreira, G. G. (2016). Avaliação in vitro de efeitos anti-inflamatórios de extratos de Pouteria torta (mart.) Radlk e Pouteria ramiflora (Mart.) Radlk.

Ferrario, C. M., \& Mullick, A. E. (2017). Renin angiotensin aldosterone inhibition in the treatment of cardiovascular disease. Pharmacological research, 125, 57-71.

Forrester, S. J., Booz, G. W., Sigmund, C. D., Coffman, T. M., Kawai, T., Rizzo, V., \& Eguchi, S. (2018). Angiotensin II signal transduction: an update on mechanisms of physiology and pathophysiology. Physiological reviews, 98(3), 1627-1738.

Hao, Q., Dong, X., Chen, X., Yan, F., Wang, X., Shi, H., \& Dong, B. (2018). Angiotensin-Converting Enzyme 2 Inhibits Angiotensin II-Induced Abdominal Aortic Aneurysms in Mice. Human gene therapy, 29(12), 1387-1395.

Hrenak, J., Paulis, L., \& Simko, F. (2016). Angiotensin A/Alamandine/MrgD axis: another clue to understanding cardiovascular pathophysiology. International journal of molecular sciences, 17(7), 1098.

Liao, W., Fan, H., Davidge, S. T., \& Wu, J. (2019). Egg white-derived antihypertensive peptide IRW (Ile-Arg-Trp) reduces blood pressure in spontaneously hypertensive rats via the ACE2/ang (1-7)/mas receptor Axis. Molecular nutrition \& food research, 63(9), 1900063.

Meems, L. M., Andersen, I. A., Pan, S., Harty, G., Chen, Y., Zheng, Y., \& Burnett Jr, J. C. (2019). Design, synthesis, and actions of an innovative bispecific designer peptide: NPA7. Hypertension, 73(4), 900-909.

Mendoza-Torres, E., Oyarzún, A., Mondaca-Ruff, D., Azocar, A., Castro, P. F., Jalil, J. E., \& Ocaranza, M. P. (2015). ACE2 and vasoactive peptides: novel players in cardiovascular/renal remodeling and hypertension. Therapeutic advances in cardiovascular disease, 9(4), 217-237.

Merz, A. A., \& Cheng, S. (2016). Sex differences in cardiovascular ageing. Heart, 102(11), 825-831.

Moher, D., Liberati, A., Tetzlaff, \& Altman. (2015). Principais itens para relatar Revisões sistemáticas e Meta-análises: A recomendação PRISMA. Epidemiologia e Serviços de Saúde, 24(2), 335-342. https://doi.org/10.5123/s1679-49742015000200017

Montezano, A. C., Cat, A. N. D., Rios, F. J., \& Touyz, R. M. (2014). Angiotensin II and vascular injury. Current hypertension reports, $16(6), 431$.

Organização Pan-Americana da Saúde e Organização Mundial da Saúde. Doenças cardiovasculares: Folha informativa. OPAS. Disponível em:https://www.paho.org/bra/index.php?option=com_content\&view=article\&id=5253:doencas-cardiovasculares\&Itemid=1096. Recuperado em: 26 jul. 2020.

Patel, V. B., Takawale, A., Ramprasath, T., Das, S. K., Basu, R., Grant, M. B., \& Oudit, G. Y. (2015). Antagonism of angiotensin 1-7 prevents the therapeutic effects of recombinant human ACE2. Journal of molecular medicine, 93(9), 1003-1013.

Patel, S. N., Ali, Q., Samuel, P., Steckelings, U. M., \& Hussain, T. (2017). Angiotensin II type 2 receptor and receptor mas are colocalized and functionally interdependent in obese zucker rat kidney. Hypertension, 70(4), 831-838.

Pinter, M., \& Jain, R. K. (2017). Targeting the renin-angiotensin system to improve cancer treatment: Implications for immunotherapy. Science Translational Medicine, 9(410).

Povlsen, A. L., Grimm, D., Wehland, M., Infanger, M., \& Krüger, M. (2020). The vasoactive Mas receptor in essential hypertension. Journal of Clinical Medicine, 9(1), 267

Qaradakhi, T., Gadanec, L. K., McSweeney, K. R., Tacey, A., Apostolopoulos, V., Levinger, I., \& Zulli, A. (2020). The potential actions of angiotensin-converting enzyme II (ACE2) activator diminazene aceturate (DIZE) in various diseases. Clinical and Experimental Pharmacology and Physiology, 47(5), 751-758.

Santos, R. A. S., Sampaio, W. O., Alzamora, A. C., Motta-Santos, D., Alenina, N., Bader, M., \& Campagnole-Santos, M. J. (2018). The ACE2/angiotensin-(17)/MAS axis of the renin-angiotensin system: focus on angiotensin-(1-7). Physiological reviews, 98(1), 505-553.

South, A. M., Shaltout, H. A., Washburn, L. K., Hendricks, A. S., Diz, D. I., \& Chappell, M. C. (2019). Fetal programming and the angiotensin-(1-7) axis: a review of the experimental and clinical data. Clinical Science, 133(1), 55-74.

Stegbauer, J., Thatcher, S. E., Yang, G., Bottermann, K., Rump, L. C., Daugherty, A., \& Cassis, L. A. (2019). Mas receptor deficiency augments angiotensin II-induced atherosclerosis and aortic aneurysm ruptures in hypercholesterolemic male mice. Journal of vascular surgery, 70(5), 1658-1668.

Te Riet, L., van Esch, J. H., Roks, A. J., van den Meiracker, A. H., \& Danser, A. J. (2015). Hipertensão: alterações do sistema renina-angiotensina-aldosterona. Circulation research, 116 (6), 960-975.

Tobón-Arroyave, S. I., Hurtado-García, P., García-Quintero, O. D., Isaza-Guzmán, D. M., \& Flórez-Moreno, G. A. (2014). Immunoexpression of NF-кB and their inhibitory subunits $\mathrm{IKB}_{\alpha} \alpha$ and $\mathrm{IkB} \beta$ in giant cell lesions of the jaws: implications for their clinical behavior. Journal of Oral Pathology \& Medicine, 44(9), $752-760$. 
Research, Society and Development, v. 10, n. 3, e44410313553, 2021

(CC BY 4.0) | ISSN 2525-3409 | DOI: http://dx.doi.org/10.33448/rsd-v10i3.13553

Tóth, A. D., Turu, G., Hunyady, L., \& Balla, A. (2018). Novel mechanisms of G-protein-coupled receptors functions: AT1 angiotensin receptor acts as a signaling hub and focal point of receptor cross-talk. Best Practice \& Research Clinical Endocrinology \& Metabolism, 32(2), 69-82.

Tufik, S., Pires, G. N., Kim, L. J., Tempaku, P., Albuquerque, R., \& Andersen, M. L. (2017). Revisão sistemática sobre a epidemiologia das doenças cardiovasculares e respiratórias e suas associações com a poluição do ar em Vitória/ES. Clinical \& Biomedical Research, 37(2).

Wang, J., He, W., Guo, L., Zhang, Y., Li, H., Han, S., \& Shen, D. (2017). The ACE2-Ang (1-7)-Mas receptor axis attenuates cardiac remodeling and fibrosis in post-myocardial infarction. Molecular medicine reports, 16(2), 1973-1981.

Wang, D., Chai, X. Q., Magnussen, C. G., Zosky, G. R., Shu, S. H., Wei, X., \& Hu, S. S. (2019). Renin-angiotensin-system, a potential pharmacological candidate, in acute respiratory distress syndrome during mechanical ventilation. Pulmonary pharmacology \& therapeutics, $58,101833$.

Zhang, W., Song, M., Qu, J., \& Liu, G. H. (2018). Epigenetic modifications in cardiovascular aging and diseases. Circulation research, 123(7), 773-786. 